\title{
A first in man phase I trial of the oral immunomodulator, indoximod, combined with docetaxel in patients with metastatic solid tumors
}

\author{
Hatem H. Soliman ${ }^{1}$, Erica Jackson ${ }^{2}$, Tony Neuger ${ }^{1}$, E. Claire Dees ${ }^{3}$, R. Donald \\ Harvey4, Hyo Han ${ }^{1}$, Roohi Ismail-Khan ${ }^{1}$, Susan Minton ${ }^{1}$, Nicholas N. Vahanian ${ }^{5}$, \\ Charles Link ${ }^{5}$, Daniel M. Sullivan' ${ }^{1}$, Scott Antonia ${ }^{1}$
}

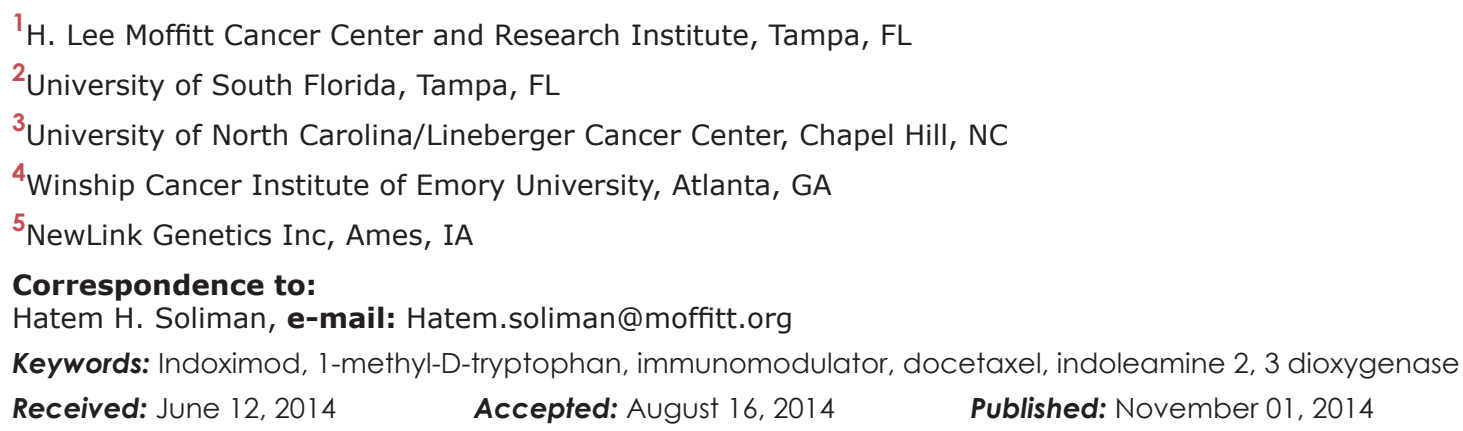

\section{ABSTRACT}

Background: Indoleamine 2,3-dioxygenase (IDO) is an enzyme that tumors use to create a state of immunosuppression. Indoximod is an IDO pathway inhibitor. Preclinical studies demonstrated that indoximod combined with chemotherapy was synergistic in a mouse model of breast cancer. A phase I 3+3 trial was designed to study the combination of docetaxel and indoximod.

Methods: Docetaxel was administered at $60 \mathrm{mg} / \mathrm{m}^{2}$ intravenously every 3 weeks dose levels $1-4$ and $75 \mathrm{mg} / \mathrm{m}^{2}$ for dose level 5 . Indoximod was given at 300,600 , 1000, 2000, and $1200 \mathrm{mg}$ PO twice daily continuously for levels 1-5, respectively. Serum drug levels were measured.

Results: Twenty-seven patients were treated, with 22 evaluable for response. DLTs included grade 3 dehydration (level 1), hypotension(level 4), mucositis (level 4) and grade 5 enterocolitis (level 2). Dose level 5 is the recommended phase II dose. The most frequent adverse events were fatigue (58.6\%), anemia $(51.7 \%)$, hyperglycemia $(48.3 \%)$, infection $(44.8 \%)$, and nausea $(41.4 \%)$. There were 4 partial responses ( 2 breast, 1 NSCLC, 1 thymic tumor). No drug-drug interactions were noted.

Conclusions: Docetaxel plus indoximod was well tolerated with no increase in expected toxicities or pharmacokinetic interactions. It was active in a pretreated population of patients with metastatic solid tumors.

\section{INTRODUCTION}

Indoleamine 2,3-dioxgenase (IDO) is a tryptophancatabolizing enzyme that inhibits the immune response. IDO was first discovered as an enzyme of the placenta, essential for the prevention of fetal rejection by maternal $\mathrm{T}$ cells [1]. It was later determined that IDO played a critical role in the modulation of autoimmunity and transplantation [2]. Because $\mathrm{T}$ cells are sensitive to tryptophan deficiency, depletion causes growth arrest in the G1 phase of the cell cycle [3, 4]. IDO can deplete the local tryptophan supply, thereby blocking the proliferation of reactive T lymphocytes $[3,4]$.

Immune cells are often present at the tumor site, not only to recognize and eliminate malignant cells, but conversely to aid the tumor in evasion of immune destruction [5]. Recent literature has determined that IDO can be used by tumor cells to avoid elimination by the host immune response [6]. It was hypothesized that an inhibitor of IDO would increase the effectiveness of the T-cell 
response against tumors leading to growth inhibition. Indoximod (D-1-methyl-tryptophan) was developed as an inhibitor of the IDO pathway. The proposed mechanism of action is reversal of the downstream effects of IDO activation through amino acid sensing and mTOR pathways [7]. Preclinical data support the ability of indoximod to reverse IDO-mediated immune suppression [8]. The immunosuppressive activity of IDO leads to an increase in the number of T-regulatory cells, as measured by their Foxp3+/CD4+/CD25+ phenotype. Indoximod has also been shown to reduce the number of T-regulatory cells [9].

In a study with MMTV-Neu mice, researchers looked at the activity of indoximod with and without paclitaxel [10]. Mice with palpable tumors were treated with control vehicle, indoximod, paclitaxel, or indoximod with paclitaxel. A taxane was chosen based on evidence that it increased effector T cells at the tumor site [11]. The combination produced significantly more tumor regression than either agent alone. This synergistic effect was lost when the experiment was repeated in immunodeficient mice, demonstrating that the benefit was dependent on an anti-tumor immune response. Additional preclinical data suggested that indoximod can synergize with other chemotherapy agents, such as doxorubicin and platinum salts [10]. The single-agent phase I trial of indoximod demonstrated very good oral bioavailability and a mild toxicity profile with no significant myelosuppression, and no maximally tolerated dose was identified up to $2000 \mathrm{mg}$ orally twice daily [12]. Based on the preclinical data and good safety profile of single-agent indoximod, we initiated a phase I trial to investigate the combination therapy of docetaxel plus indoximod in patients with metastatic solid tumors.

\section{PATIENTS AND METHODS}

\section{Patient eligibility}

Eligibility for this study included patients with advanced solid tumors, age greater than 18 years, life expectancy greater than 4 months, Eastern Cooperative Oncology Group (ECOG) performance status 0-2, and adequate organ/marrow function. Patients were excluded if they met any of the following criteria: 1) chemotherapy/ radiotherapy within the past 3 weeks, 2) untreated brain metastases, 3) uncontrolled concurrent major illness, 4) current use or previous allergic reaction to L-tryptophan, 5) active autoimmune disease or chronic inflammatory condition requiring use of steroids or systemic immunosuppressants, 6) pregnant, 7) AIDS/ HIV infection, or 8) history of gastrointestinal disease causing malabsorption/obstruction. In addition, patients that received active immunotherapies such as adjuvant interferon less than 1 year prior to enrollment were excluded. There were no restrictions on number of prior lines of therapy, and could have received prior docetaxel in the adjuvant (but not metastatic) setting. Any patient who received prior experimental immunotherapy consisting of targeted monoclonal antibodies was excluded. However, patients who received prior therapy with approved monoclonal antibodies such as bevacizumab, cetuximab, panitumumab, or trastuzumab were eligible. Patients were accrued through the Southeast Phase 2 Consortium consisting of the following locations: H. Lee Moffitt Cancer Center and Research Institute, Billings Clinic Cancer Center, Massey Cancer Center, Lineberger Comprehensive Cancer Center, and the Winship Cancer Institute of Emory University. Both men and women and members of all races and ethnic groups were eligible for this trial.

\section{Study design}

The protocol was approved by the National Cancer Institute's Cancer Therapeutics Evaluation Program and conducted in accordance with all federal and institutional guidelines. All patients provided written informed consent under an Institutional Review Board-approved protocol prior to initiation of any study procedure (University of South Florida IRB, FWA\# FWA00001669).

The study followed a $3+3$ escalation design to determine the maximum tolerated dose (MTD). The MTD is defined as the highest dose level in which less than or equal to one out of six patients experiences a dose-limiting toxicity (DLT). DLTs were defined as 1) non-hematological grade 3 or greater toxicity probably attributable to therapy and not to underlying disease, 2) absolute neutrophil count of less than 500/ $\mathrm{mL}$ for longer than 7 days or neutropenic fever requiring hospitalization despite the use of white blood cell colonystimulating factors and dose reduction of docetaxel to $\left.60 \mathrm{mg} / \mathrm{m}^{2}, 3\right)$ grade 4 thrombocytopenia $(<25,000 / \mathrm{mL})$, and 4) dose delay due to hematological toxicity for more than 14 days. Grade 3 hypophysitis and dermatitis were the only exceptions to the DLT rule.

This phase I study evaluated the safety of docetaxel at $60-75 \mathrm{mg} / \mathrm{m}^{2}$ in combination with escalating doses of indoximod (Table 1). Dose level 5 was amended after accrual to dose level 4 was completed due to pharmacokinetic data from the monotherapy trial that revealed maximal absorption was reached at $1200 \mathrm{mg}$ PO twice daily.

Indoximod was supplied by the National Cancer Institute's Pharmacy Branch and NewLink Genetics Inc (50 mg and $200 \mathrm{mg}$ hard gelatin capsules). It was administered orally twice daily for 21-day continuous cycles on an empty stomach. Docetaxel was prepared with a single-dose diluent containing $13 \%$ ethanol in water and was then administered intravenously over 1 hour. Standard supportive medications, including dexamethasone and anti-histamines as required, were used with docetaxel. 
Table 1. Dose escalations and treatments administered

\begin{tabular}{|c|c|c|c|c|c|c|}
\hline Level & $\begin{array}{l}\text { Docetaxel } \\
\left(\mathrm{mg} / \mathrm{m}^{2}\right)\end{array}$ & $\begin{array}{l}\text { Indoximod } \\
\text { (mg) }\end{array}$ & $\begin{array}{l}\text { No. of Patients } \\
\text { Treated }\end{array}$ & $\begin{array}{l}\text { No. of Cycles } \\
\text { Administered }\end{array}$ & $\begin{array}{l}\text { Cycles per } \\
\text { Patient } \\
\text { (median) }\end{array}$ & Dose limiting toxicities \\
\hline 1 & 60 & 300 & 7 & 28 & 3 & Grade 3 dehydration \\
\hline 2 & 60 & 600 & 6 & 35 & 3 & Grade 5 colitis \\
\hline 3 & 60 & 1000 & 6 & 39 & 3 & \\
\hline 4 & 60 & 2000 & 2 & 2 & 1 & $\begin{array}{c}\text { Grade } 3 \text { hypotension, } \\
\text { mucositis }\end{array}$ \\
\hline 5 & 75 & 1200 & 6 & 16 & 2 & \\
\hline
\end{tabular}

The primary endpoint of this trial was to determine the MTD of the indoximod/docetaxel combination using Common Terminology Criteria for Adverse Events 4.0. Secondary endpoints included the determination of the pharmacokinetic data and the overall objective response rate per Response Evaluation Criteria in Solid Tumors (RECIST) 1.1 criteria.

\section{Safety evaluations}

Complete blood counts and metabolic panels were obtained at baseline and every 3 weeks. Pituitary function tests (thyroid stimulating hormone, free T4, leutinizing hormone, follicular stimulating hormone, and adrenocortical hormone) were obtained at baseline and every 6 weeks. Patients underwent complete physicals and adverse event evaluation once each cycle and as clinically indicated.

\section{Response ealuation}

Overall response rate was determined via the criteria described by the RECIST 1.1 guidelines. Baseline evaluations were conducted within 14 days prior to the start of therapy. Scans were performed within 4 weeks prior to the start of therapy. Patients were then reevaluated every 6 weeks with diagnostic CT scans. The best overall response achieved during study therapy was recorded for each patient. The response data presented herein underwent independent radiology review. The duration of overall response was measured from the time criteria were met for complete or partial response until the first date that recurrent or progressive disease was documented. In patients exhibiting response or disease stabilization, treatment was continued until 1) disease progression, 2) intercurrent illness that prevented further treatment, 3) unacceptable adverse events despite appropriate supportive care, or 4) patient withdrawal from trial.

\section{Pharmacokinetic methods}

Validated liquid chromatography triple quadrupole with tandem mass spectrometry (LC/MS/MS) methods were used to determine levels of indoximod and docetaxel in plasma. The methods were validated per ICH/FDA guidelines. Plasma samples were prepared for chromatographic injections by protein precipitation (PPT) in both instances. However, methods were conducted as separate analysis due to the vast differences in analyte characteristics.

Indoximod calibration and quality control samples were made by adding known amounts of indoximod to blank plasma. Indoximod-d3, stable isotope labeled internal standard, was used in the assay. A Sirocco 96well PPT plate (Waters Corp, Milford, MA) was utilized similar to the manufacturer's suggestion. Samples were injected into a ThermoAccela/TSQ Quantum LC/MS/ MS system (Thermo Scientific, San Jose, CA). Mobile phase consisted of water and methanol, both containing $0.1 \%$ acetic acid. Gradient pumping conditions were run, and samples were maintained at $4{ }^{\circ} \mathrm{C}$ in the autosampler during sequences. Indoximod was separated using a Luna C18 (2 x 50 mm, $3 \mu \mathrm{m}$ ) column (Phenomenex, Inc., Torrance, CA). Electrospray ionization was employed, and multiple reaction monitoring was conducted in positive mode utilizing the following molecular transitions; $219 \rightarrow 160$ and $222 \rightarrow 163$ for indoximod and indoximod-d3, respectively. Peaks were detected and integration was performed with Thermo LC Quan software.

The calibration and quality control samples for docetaxel were made in similar fashion as previous with blank plasma. Docetaxel-d9, stable isotope labeled internal standard, was utilized for this analysis. Protein precipitation was carried out in an Ostro 96-well PPT plate (Waters Corp) according to the manufacturer's suggestion. The same instrument and mobile phase was used as previously described. Samples were maintained at $8^{\circ} \mathrm{C}$ in the autosampler during sequences, and gradient pumping conditions were used. Docetaxel was separated using a Zobrax SB C18 (2.1 x 50 mm, $3.5 \mu \mathrm{m})$ column (Agilent Technologies, San Jose, CA). Electrospray ionization was utilized, and multiple reaction monitoring was conducted in negative mode tracking the following molecular transitions: $806 \rightarrow 672$ and $815 \rightarrow 672$ for docetaxel and 
docetaxel-d9, respectively. Once again, LC Quan was used for detection of peaks and integration.

Calibration curves were generated for each run, and patient sample concentrations were back-calculated from the corresponding regression line using LC Quan. The assays are linear from 5 to $2500 \mathrm{ng} / \mathrm{mL}$ for both indoximod and docetaxel. Recovery of indoximod from plasma is on average greater than $95 \%$. Inter- and intraassay variability was less than $9 \%$ with a relative mean error of less than $10 \%$ for indoximod. Docetaxel average recovery from plasma was determined to be greater than $78 \%$. Docetaxel assay inter- and intra-assay variability was less than $11 \%$ with a relative mean error of less than $6 \%$. Plasma concentration-time data for both drugs was analyzed by non-compartmental pharmacokinetic methods using Phoenix WinNonlin 6.3 (Pharsight Corp., Mountain View, CA). Data in the terminal, log-linear phase were analyzed by linear regression to estimate terminal elimination rate constant and half-life. These additional pharmacokinetic parameters were also determined: $\mathrm{AUC}_{0-48}, \mathrm{AUC}_{0 \text {-inf }}, \mathrm{C}_{\max }, \mathrm{T}_{\max }$, clearance, and volume of distribution.

\section{RESULTS}

\section{Patient population}

Patient demographics are outlined in Table 2. Patients with a variety of cancers were enrolled; however, most primary sites were categorized as non-small cell lung carcinoma (34\%) or breast (28\%). Eighty-six percent of patients received treatment in the metastatic setting before entering the study. Of the four untreated patients, one withdrew consent during cycle 1 and was unevaluable for response. One of the breast cancer patients was assigned to dose level 1 after relapsing within six months of adjuvant dose dense doxorubicin/cyclophosphamide/paclitaxel chemotherapy. The second breast patient relapsed on adjuvant endocrine therapy and was assigned to dose level 2. The fourth patient was a stage IIIB unresectable lung cancer patient who initially was treated with cisplatin/pemetrexed/vorinostat plus radiation, then had progression of disease and was assigned to dose level 3. Patients received a median of five prior therapies, mostly consisting of chemotherapy. Almost all patients were ECOG performance status 0 or 1 .

\section{Patient flow}

Thirty-three patients were screened for the study with five failing to meet all screening criteria. Twentynine patients were then registered for treatment. Two patients withdrew consent during cycle 1, with 27 patients initiating treatment. One patient began treatment, was then found to be ineligible after the first cycle, and was replaced. One patient (dose level 2) expired on cycle 1, day 10 and was not evaluable for response. Three patients withdrew consent during cycle 1 and were not assessed for the response endpoint. Twenty-two patients were included in the response analysis. Figure 1 shows a chart of patient flow through the trial.

\section{Adverse events}

Table 3 lists the frequency of any grade 3, 4, or 5 toxicities that occurred, as well as any grade 1 or 2 toxicity with a frequency greater than $25 \%$ regardless of attribution. Adverse events occurring with the highest frequency included grade 1 anemia, fatigue, and hyperglycemia $(41 \%, 45 \%$, and $38 \%$, respectively). Common grade $3 / 4$ adverse effects included neutropenia and febrile neutropenia (both 13\%).

\section{Dose-limiting toxicities}

DLTs included grade 3 dehydration at level 1, grade 5 colitis at level 2, and grade 3 hypotension and mucositis at level 4 . The grade 5 colitis was caused by sudden mesenteric ischemia leading to sepsis. The death was determined unlikely to be related to indoximod by the site investigators, as well as after thorough safety review by the protocol monitoring committee. Dose level $5\left(75 \mathrm{mg} / \mathrm{m}^{2}\right.$ of docetaxel $+1200 \mathrm{mg}$ PO twice daily of indoximod) was administered to 6 patients without a DLT and thus was deemed the maximally tolerated dose level.

\section{Response rate}

The overall objective response rates are summarized in Table 4. Four patients achieved a partial response $(18 \%)$, one patient achieved stable disease for longer than 6 months (4\%), nine patients achieved stable disease for less than 6 months (36\%), and eight patients had progressive disease $(36 \%)$.

Figure 2 is a waterfall plot of each patient's best overall response. Four breast cancer patients achieved a reduction in tumor burden $(64.8 \%, 33 \%, 14.6 \%$, and $6.2 \%$ reduction of target lesions). The best of these responders was the untreated breast cancer patient in dose level two who had received only adjuvant endocrine therapy prior to study entry. Two non-small cell lung cancer patients showed a reduction in tumor size $(56.5 \%$ and $8.2 \%)$, as well as one patient with thymic cancer (78.3\% reduction). The durations of the objective responses were 15.4 and 5.9 months in the two breast cancer patients. In the lung cancer and thymic cancer patients the duration of response was 7.1 and 5.9 months respectively. It should be noted that the thymic cancer patient discontinued therapy secondary to fatigue and not due to confirmed radiographic progression of his disease. None of the objective responders were previously treated with docetaxel. Five patients showed only a slight increase in tumor burden with less than $10 \%$ growth of target lesions. 
Table 2. Patient and Treatment Characteristics $(\mathbf{N}=\mathbf{2 9})$

\begin{tabular}{|c|c|}
\hline Demographic & \\
\hline Sex, n (\%) & \\
\hline Men & $15(51)$ \\
\hline Women & $14(49)$ \\
\hline Age & \\
\hline Mean $\pm \mathrm{SD}$ & $53+/-10$ \\
\hline Median (range) & 53 \\
\hline Ethnicity, n (\%) & \\
\hline White & $25(86)$ \\
\hline Black & $3(10)$ \\
\hline Other & $1(3)$ \\
\hline Disease type, n (\%) & \\
\hline NSCLC & $10(34)$ \\
\hline Breast (4 ER+, 4 TNBC) & $8(28)$ \\
\hline Laryngeal & $2(7)$ \\
\hline Esophageal & $2(7)$ \\
\hline Ovarian & $2(7)$ \\
\hline Uterine & $1(3)$ \\
\hline Thymus & $1(3)$ \\
\hline Liposarcoma & $1(3)$ \\
\hline Rectal & $1(3)$ \\
\hline Pancreas & $1(3)$ \\
\hline ECOG Performance Status & \\
\hline 0 & $11(37)$ \\
\hline 1 & $14(48)$ \\
\hline 2 & $4(13)$ \\
\hline Previous therapies (median & \\
\hline Total & 5 \\
\hline Chemotherapy & 3 \\
\hline Previous therapy, n (\%) & \\
\hline Total & $25(86)$ \\
\hline Chemotherapy & $24(83)$ \\
\hline Radiation & $9(31)$ \\
\hline Hormonal/endocrine & $5(17)$ \\
\hline Trastuzumab & $2(7)$ \\
\hline
\end{tabular}

\section{Pharmacokinetics}

Indoximod pharmacokinetics were similar to results previously reported by our group in the phase I single-agent trial [12]. The half-life was $11.3( \pm 5.0)$ hours $(n=23)$ and AUC was proportional to dose. When comparing day 1 and 8 results, the pharmacokinetics for indoximod were consistent, indicating little to 


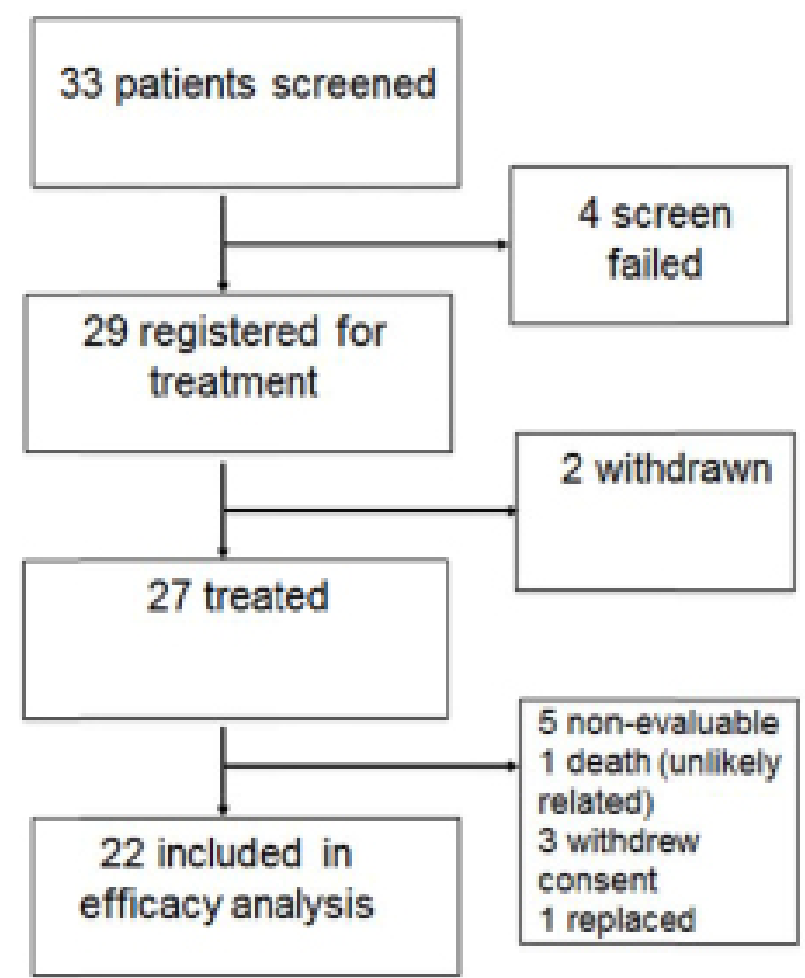

Figure 1: Patient flow diagram

no interaction with docetaxel. When comparing AUC(0-inf) on day 1 with $\mathrm{AUC}(\mathrm{ss})$ on day 8 , results were nearly the same. Figure 3 represents AUC comparisons. The Cmax of indoximod on day 8 was higher than on day 1 at two dose levels (600 and $1000 \mathrm{mg}$ ), but the standard deviation among the patients may have contributed. Doseindependent pharmacokinetic parameter estimates for indoximod can be found in Table 5 .

The pharmacokinetics results for docetaxel do not appear to be affected by indoximod. The results of the two dose levels evaluated were similar to results reported in single-agent studies [13]. Dose was proportional to AUC and Cmax for the study population. Half-life reported here for the entire population, $20.9( \pm 7.8)$ hours $(n=23)$, was also similar to previous evaluations. Docetaxel pharmacokinetics by dose level can be found in Table 6 .

\section{DISCUSSION}

The field of cancer immunotherapy is undergoing a renaissance due to a greater understanding of the complex regulatory pathways that cause tumor-related immunosuppression. Checkpoint inhibitors such as ipilimumab and nivolimumab demonstrated dramatic responses and durable long-term disease control in a fraction of patients with melanoma and non-small cell lung cancer [14-16]. To increase the clinical benefit to patients, combination strategies will need to be explored as a way to overcome immune suppression mediated by other pathways. Indoleamine 2,3 dioxygenase represents one such pathway, and there are efforts underway to see how IDO inhibition can be used to boost the efficacy of other immunotherapies [17]. Another important consideration is that the efficacy of some chemotherapeutics and targeted monoclonal antibodies is in part dependent on their ability to promote an effective anti-tumor response [18-21]. Therefore, combining immunomodulators with chemotherapy or targeted agents to boost this immune response is a logical next step.

This phase I trial provides important information on the feasibility and safety of this chemoimmunotherapy approach using a taxane and an IDO pathway inhibitor. The multiple indications of docetaxel will allow this combination to be tested in a variety of solid tumors that overexpress IDO, such as breast cancer, non-small cell lung cancer, prostate cancer, gastric cancer, and oropharyngeal cancer [8]. Indoximod appears to be ideal from a safety standpoint in that it does not add significantly to the toxicity burden or myelosuppression imposed by the chemotherapy agent. Nadir counts were not routinely obtained due to the high baseline incidence of neutropenia $(>90 \%)$ from single-agent docetaxel, which would make it difficult to ascertain the impact of indoximod on asymptomatic neutropenia. This is especially the 
Table 3. Adverse events by grade regardless of attribution

\begin{tabular}{|c|c|c|c|c|c|}
\hline Toxicity & Grade 1 n(\%) & Grade 2 n(\%) & Grade 3 n(\%) & Grade 4 n(\%) & Grade 5 n(\%) \\
\hline \multicolumn{6}{|l|}{ Hematologic } \\
\hline Anemia & $12(41)$ & $8(28)$ & 1(3) & 0 & 0 \\
\hline Leukopenia & 0 & 0 & 1(3) & 2(7) & 0 \\
\hline Lymphopenia & 0 & 0 & $2(7)$ & 0 & 0 \\
\hline Neutropenia & 0 & 0 & 1(3) & $3(10)$ & 0 \\
\hline Thrombocytopenia & 0 & 0 & 1(3) & 0 & 0 \\
\hline \multicolumn{6}{|l|}{ Endocrine } \\
\hline Hyperglycemia & $11(38)$ & $8(28)$ & 1(3) & 0 & 0 \\
\hline \multicolumn{6}{|l|}{ Infection } \\
\hline Febrile Neutropenia & 0 & 0 & $4(14)$ & 0 & 0 \\
\hline GI Infection & 0 & 0 & $1(3)$ & 0 & 0 \\
\hline Gram Negative Infection & 0 & 0 & 1(3) & 0 & 0 \\
\hline Pneumonia & 0 & 0 & $2(7)$ & 0 & 0 \\
\hline Sepsis & 0 & 0 & 0 & 1(3) & 0 \\
\hline Skin Infection & 0 & 0 & 1(3) & 0 & 0 \\
\hline Tooth Abscess & 0 & 0 & 1(3) & 0 & 0 \\
\hline \multicolumn{6}{|l|}{ Gastrointestinal } \\
\hline Abdominal Pain & 0 & 0 & $1(3)$ & 0 & 0 \\
\hline Anorexia & $8(28)$ & 0 & 0 & 0 & 0 \\
\hline Bowel Perforation & 0 & 0 & 0 & 1(3) & 0 \\
\hline Colitis & 0 & 0 & 0 & 0 & $1(3)^{*}$ \\
\hline Constipation & $8(28)$ & 0 & 0 & 0 & 0 \\
\hline Dehydration & 0 & 0 & $2(7)^{*}$ & 0 & 0 \\
\hline Diarrhea & $8(28)$ & 0 & 0 & 0 & 0 \\
\hline Nausea & $10(34)$ & 0 & 1(3) & 0 & 0 \\
\hline Oral Mucositis & 0 & 0 & $1(3)^{*}$ & 0 & 0 \\
\hline Vomiting & $8(28)$ & 0 & 0 & 0 & 0 \\
\hline \multicolumn{6}{|l|}{ Metabolic } \\
\hline Hypercalcemia & 0 & 0 & 1(3) & 0 & 0 \\
\hline Hypoalbuminemia & $9(31)$ & 0 & $2(7)$ & 0 & 0 \\
\hline Hypocalcemia & 0 & 0 & 1(3) & 1(3) & 0 \\
\hline Hypokalemia & 0 & 0 & 1(3) & 0 & 0 \\
\hline Hyponatremia & 0 & 0 & $4(14)$ & 0 & 0 \\
\hline \multicolumn{6}{|l|}{ Pulmonary } \\
\hline Cough & $6(21)$ & 0 & 0 & 0 & 0 \\
\hline Dyspnea & $8(28)$ & 0 & 1(3) & 0 & 0 \\
\hline Pleuritic Chest Pain & 0 & 0 & 1(3) & 0 & 0 \\
\hline
\end{tabular}

(Continued) 


\begin{tabular}{|c|c|c|c|c|c|}
\hline Toxicity & Grade 1 n(\%) & Grade 2 n(\%) & Grade 3 n(\%) & Grade 4 n(\%) & Grade 5 n(\%) \\
\hline \multicolumn{6}{|l|}{ Renal } \\
\hline Increased Creatinine & 0 & 0 & 1(3) & 0 & 0 \\
\hline Hematuria & 0 & 0 & 1(3) & 0 & 0 \\
\hline \multicolumn{6}{|l|}{ Vascular } \\
\hline Hypotension & 0 & 0 & $3(10)^{*}$ & 0 & 0 \\
\hline \multicolumn{6}{|l|}{ Muskuloskeletal } \\
\hline Alopecia & $8(28)$ & 0 & 0 & 0 & 0 \\
\hline Arthralgia & 0 & 0 & 1(3) & 0 & 0 \\
\hline Bone Pain & 0 & 0 & 1(3) & 0 & 0 \\
\hline \multicolumn{6}{|l|}{ Neurologic } \\
\hline Peripheral Neuropathy & $7(24)$ & 0 & 1(3) & 0 & 0 \\
\hline Weakness & 0 & 0 & 1(3) & 0 & 0 \\
\hline \multicolumn{6}{|c|}{ Constitutional Symptoms } \\
\hline Fatigue & $13(45)$ & $13(45)$ & 0 & 0 & 0 \\
\hline \multicolumn{6}{|l|}{ Pain } \\
\hline Headache & 0 & 0 & 2(7) & 0 & 0 \\
\hline Jaw Pain & 0 & 0 & $1(3)$ & 0 & 0 \\
\hline \multicolumn{6}{|l|}{ General Disorders } \\
\hline Multi-Organ Failure & 0 & 0 & 0 & 1(3) & 1(3) \\
\hline
\end{tabular}

Table 4. Overall response rate in 22 patients included in efficacy analysis

\begin{tabular}{|l|l|}
\hline Objective Response Rate & \multicolumn{1}{l|}{ Number of Patients, $\mathbf{n}(\%)$} \\
\hline Complete response & 0 \\
\hline Partial response & $4(18)$ \\
\hline Stable disease $>6$ months & $1(4)$ \\
\hline Stable disease $<6$ months & $9(40)$ \\
\hline Progressive disease & $8(36)$ \\
\hline
\end{tabular}

case in a smaller phase I, pretreated patient population with diminished bone marrow reserves. Therefore, our safety evaluations focused on clinically relevant febrile neutropenic events or prolonged myelosuppression that would delay subsequent therapy. The febrile neutropenia rate of $14 \%$ was not significantly greater than what has been described in phase III trials, but it should be noted that these historical rates were shown in first-line metastatic patients [22].

The challenge of fully assessing increased toxicity from an investigational compound added to an agent such as docetaxel within a small phase I trial is difficult.
However, our ongoing randomized phase II combination trial in first-line metastatic breast cancer patients incorporates interim safety analyses with early stopping rules for excessive toxicity. This will allow a more adequately powered and meaningful assessment regarding whether the combination of docetaxel and indoximod is more toxic than docetaxel monotherapy. As far as the activity of the combination is concerned, any definitive comparative interpretation between the combination and historical activity of docetaxel monotherapy is not possible in this small, heterogeneous group of patients. The fact that 4 of the 8 breast cancer patients showed some degree 


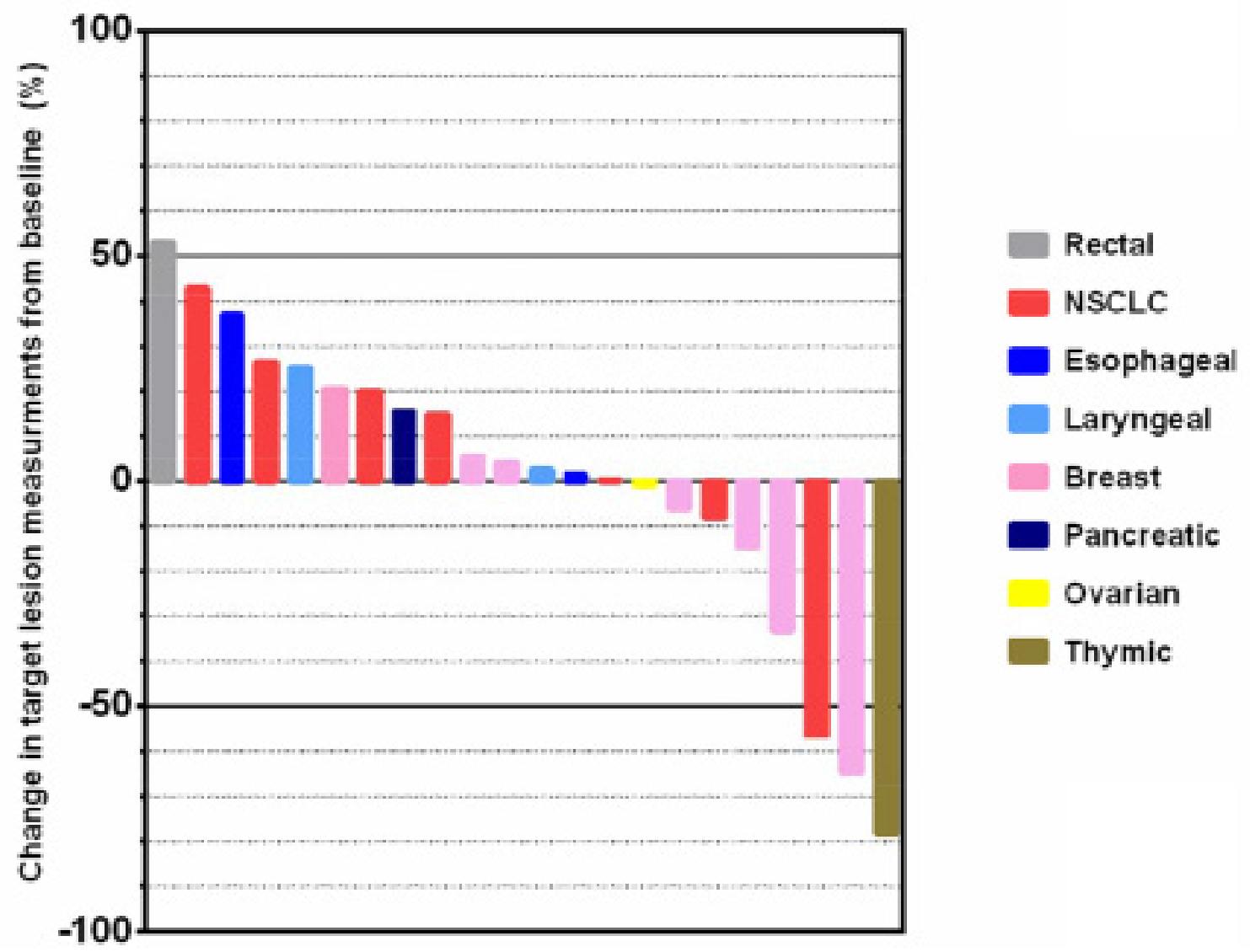

Figure 2: A waterfall plot demonstrating the greatest extent of target lesion measurement changes expressed as a percentage of the baseline measurement.

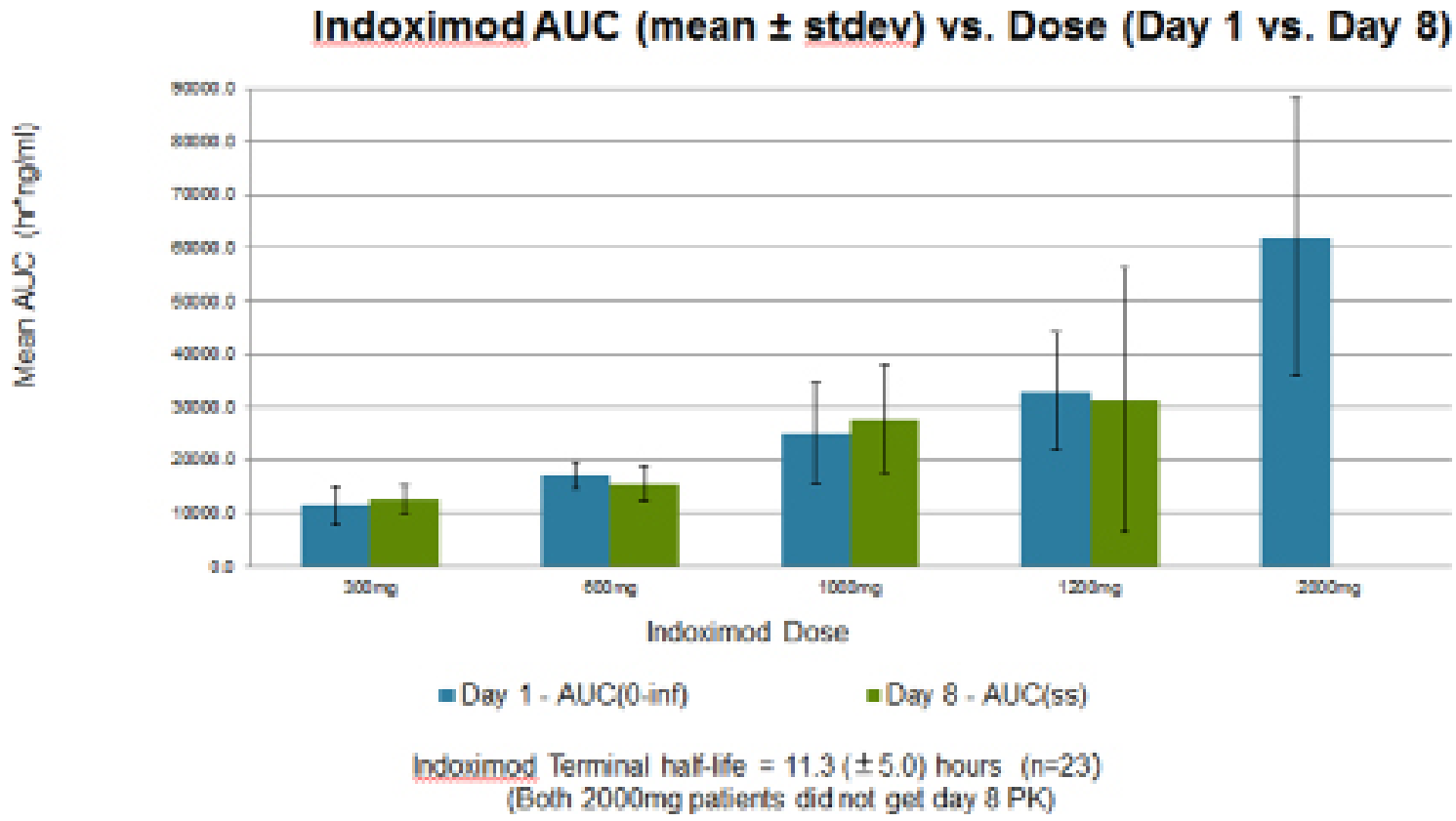

Figure 3: Mean area under the curve (AUC) values of indoximod on cycle 1 days 1 (blue bars) and 8 (green bars) across the five dose levels of indoximod on the $\mathbf{x}$ axis. Docetaxel did not significantly affect indoximod AUC values at steady state (ss) across all dose levels. 
Table 5. Indoximod dose-independent pharmacokinetics, day 1 vs. day 8

Tmax, hours

Vol. of Distribution, L Clearance, L/hour

\begin{tabular}{|l|l|l|l|l|}
\hline Day 1 & $(\mathrm{n}=23)$ & $3.6( \pm 2.0)$ & $634.4( \pm 463.1)$ & $37.2( \pm 14.4)$ \\
\hline Day 8 & $(\mathrm{n}=19)$ & $3.3( \pm 1.9)$ & $358.7( \pm 326.5)$ & $43.9( \pm 42.1)$ \\
\hline
\end{tabular}

Table 6. Docetaxel pharmacokinetics by dose level on cycle 1 , day 1

t1/2, hours Cmax, ng/mL AUC(0-48hr), AUC(0-INF), Vol. of $\quad$ Clearance,

hr*ng/mL hr*ng/mL Distribution, $L \quad$ L/hour

\begin{tabular}{|l|l|l|l|l|l|l|l|}
\hline $\mathbf{6 0} \mathbf{~ m g} / \mathbf{m}^{2}$ & $(\mathrm{n}=20)$ & $19.9 \pm 7.4$ & $1660.7 \pm 1123.8$ & $3634.9 \pm 2487.2$ & $4082.4 \pm 2611.8$ & $1145.5 \pm 811.4$ & $38.7 \pm 26.0$ \\
\hline $\mathbf{7 5} \mathbf{~ m g} / \mathbf{m}^{2}$ & $(\mathrm{n}=3)$ & $28.1 \pm 7.7$ & $1974.6 \pm 655.0$ & $3842.6 \pm 2299.1$ & $4631.2 \pm 2498.5$ & $1805.1 \pm 999.6$ & $43.1 \pm 20.2$ \\
\hline
\end{tabular}

of anti-tumor activity is an encouraging preliminary signal and warrants further investigation.

A final point that merits discussion is the rationale for the dose selection. While there is a slight increase in the response rate between $75 \mathrm{mg} / \mathrm{m}^{2}$ and $100 \mathrm{mg} / \mathrm{m}^{2}$ docetaxel in breast cancer patients, there is no significant difference in time to progression between the two doses [22]. Also, the higher toxicity of the $100 \mathrm{mg} / \mathrm{m}^{2}$ dose makes it difficult to administer in metastatic patients in combination with other investigational agents [23]. Many combination trials have used the $75 \mathrm{mg} / \mathrm{m}^{2}$ docetaxel dose as their preferred recommended phase II/III dose in patients with metastatic breast cancer for these reasons [24, 25]. A single 1200 $\mathrm{mg}$ dose of indoximod almost totally saturates the gut, and higher doses do not significantly increase peak serum levels. This serves as our justification for moving forward with the recommended phase II dose of $75 \mathrm{mg} /$ $\mathrm{m}^{2}$ of docetaxel in combination with twice daily 1200 $\mathrm{mg}$ of indoximod. A limitation of this data from a small phase 1 trial is that one cannot exclude the possibility that indoximod could exert better immunomodulatory effects at slightly higher or lower dose levels of docetaxel.

In summary, we found encouraging activity in a pre-treated population of patients with metastatic disease. According to the pharmacokinetic data, there were no drug-drug interactions between indoximod and docetaxel. The combination was well tolerated with a toxicity profile similar to docetaxel alone. The recommended phase II dose is $75 \mathrm{mg} / \mathrm{m}^{2}$ of docetaxel every 3 weeks in combination with $1200 \mathrm{mg}$ indoximod PO twice daily. The response and safety data support the conclusion that this combination is feasible to administer and should be investigated further in a larger randomized phase II trial. A randomized, double blind, placebocontrolled phase II study of docetaxel plus indoximod in metastatic breast cancer patients (NLG201) is now accruing patients.

\section{ACKNOWLEDGEMENTS}

We gratefully acknowledge the efforts of the DCTD CTEP program at the NCI including Drs. Howard Streicher and Jaime Zwiebel in their support of this trial. We also appreciate the work of Dr. Jay Ramsey from NewLink in providing support for the analysis of the data and study drug supply. Thanks to Dr. Richard Lush for his supervision over the translational research core/ pharmacokinetics facility at the Moffitt Cancer Center. Finally, thanks to Dr. John Kauh from Emory for his patient recruitment at the Emory Winship Cancer Institute.

This trial was conducted by the Southeast Phase 2 N01 Consortium under NCI contract \#HHSN261201100100C.

\section{REFERENCES}

1. Munn DH, Zhou M, Attwood JT, Bondarev I, Conway SJ, Marshall B, Brown C, Mellor AL. Prevention of allogeneic fetal rejection by tryptophan catabolism. Science. 1998; 281:1191-1193.

2. Mellor AL, Munn DH. Creating immune privilege: active local suppression that benefits friends, but protects foes. Nature reviews Immunology. 2008; 8:74-80.

3. Munn DH, Shafizadeh E, Attwood JT, Bondarev I, Pashine A, Mellor AL. Inhibition of T cell proliferation by macrophage tryptophan catabolism. The Journal of experimental medicine. 1999; 189:1363-1372.

4. Grohmann U, Fallarino F, Puccetti P. Tolerance, DCs and tryptophan: much ado about IDO. Trends in immunology. 2003; 24:242-248.

5. Hanahan D, Weinberg RA. Hallmarks of cancer: the next generation. Cell. 2011; 144:646-674.

6. Friberg $M$, Jennings $R$, Alsarraj M, Dessureault S, Cantor A, Extermann M, Mellor AL, Munn DH, Antonia SJ. 
Indoleamine 2,3-dioxygenase contributes to tumor cell evasion of $\mathrm{T}$ cell-mediated rejection. International journal of cancer Journal international du cancer. 2002; 101:151-155.

7. Metz R, Rust S, Duhadaway JB, Mautino MR, Munn DH, Vahanian NN, Link CJ, Prendergast GC. IDO inhibits a tryptophan sufficiency signal that stimulates mTOR: A novel IDO effector pathway targeted by D-1-methyltryptophan. Oncoimmunology. 2012; 1:1460-1468.

8. Uyttenhove C, Pilotte L, Theate I, Stroobant V, Colau D, Parmentier N, Boon T, Van den Eynde BJ. Evidence for a tumoral immune resistance mechanism based on tryptophan degradation by indoleamine 2,3-dioxygenase. Nature medicine. 2003; 9:1269-1274.

9. Munn DH. Blocking IDO activity to enhance anti-tumor immunity. Frontiers in bioscience. 2012; 4:734-745.

10. Muller AJ, DuHadaway JB, Donover PS, Sutanto-Ward E, Prendergast GC. Inhibition of indoleamine 2,3-dioxygenase, an immunoregulatory target of the cancer suppression gene Bin1, potentiates cancer chemotherapy. Nature medicine. 2005; 11:312-319.

11. Mason K, Staab A, Hunter N, McBride W, Petersen S, Terry N, Milas L. Enhancement of tumor radioresponse by docetaxel: Involvement of immune system. International journal of oncology. 2001; 18:599-606.

12. Soliman HH, Neuger A, Noyes D, Vahanian NN, Link CJ, Munn D, Streicher H, Sullivan D, Antonia S. A phase I study of 1-methyl-D-tryptophan in patients with advanced malignancies. J Clin Oncol. 2012; 30.

13. Bruno R, Hille D, Riva A, Vivier N, ten Bokkel Huinnink WW, van Oosterom AT, Kaye SB, Verweij J, Fossella FV, Valero V, Rigas JR, Seidman AD, Chevallier B, Fumoleau P, Burris HA, Ravdin PM, et al. Population pharmacokinetics/pharmacodynamics of docetaxel in phase II studies in patients with cancer. J Clin Oncol. 1998; 16:187-196.

14. Page DB, Postow MA, Callahan MK, Wolchok JD. Checkpoint modulation in melanoma: an update on ipilimumab and future directions. Current oncology reports. 2013; 15:500-508.

15. Robert C, Thomas L, Bondarenko I, O'Day S, M DJ, Garbe C, Lebbe C, Baurain JF, Testori A, Grob JJ, Davidson N, Richards J, Maio M, Hauschild A, Miller WH Jr., Gascon P, et al. Ipilimumab plus dacarbazine for previously untreated metastatic melanoma. The New England journal of medicine. 2011; 364:2517-2526.

16. Sznol M, Chen L. Antagonist antibodies to PD-1 and B7-H1 (PD-L1) in the treatment of advanced human cancer--response. Clinical cancer research: an official journal of the American Association for Cancer Research. $2013 ; 19: 5542$.

17. Holmgaard RB, Zamarin D, Munn DH, Wolchok JD, Allison JP. Indoleamine 2,3-dioxygenase is a critical resistance mechanism in antitumor $\mathrm{T}$ cell immunotherapy targeting CTLA-4. The Journal of experimental medicine. 2013; 210:1389-1402.

18. Ghiringhelli F, Apetoh L. The interplay between the immune system and chemotherapy: emerging methods for optimizing therapy. Expert review of clinical immunology. 2014; 10:19-30.

19. Ladoire S, Arnould L, Mignot G, Apetoh L, Rebe C, Martin F, Fumoleau P, Coudert B, Ghiringhelli F. T-bet expression in intratumoral lymphoid structures after neoadjuvant trastuzumab plus docetaxel for HER2-overexpressing breast carcinoma predicts survival. British journal of cancer. 2011; 105:366-371.

20. Ladoire S, Mignot G, Dabakuyo S, Arnould L, Apetoh L, Rebe C, Coudert B, Martin F, Bizollon MH, Vanoli A, Coutant C, Fumoleau P, Bonnetain F, Ghiringhelli F. In situ immune response after neoadjuvant chemotherapy for breast cancer predicts survival. The Journal of pathology. $2011 ; 224: 389-400$.

21. Zitvogel L, Apetoh L, Ghiringhelli F, Andre F, Tesniere A, Kroemer G. The anticancer immune response: indispensable for therapeutic success?. The Journal of clinical investigation. 2008; 118:1991-2001.

22. Harvey V, Mouridsen H, Semiglazov V, Jakobsen E, Voznyi E, Robinson BA, Groult V, Murawsky M, Cold S. Phase III trial comparing three doses of docetaxel for second-line treatment of advanced breast cancer. J Clin Oncol. 2006; 24:4963-4970.

23. Salminen E, Bergman M, Huhtala S, Ekholm E. Docetaxel: standard recommended dose of $100 \mathrm{mg} / \mathrm{m}(2)$ is effective but not feasible for some metastatic breast cancer patients heavily pretreated with chemotherapy-A phase II singlecenter study. J Clin Oncol. 1999; 17:1127.

24. Schwartzberg LS, Badarinath S, Keaton MR, Childs BH. Phase II Multicenter Study of Docetaxel and Bevacizumab With or Without Trastuzumab as First-Line Treatment for Patients With Metastatic Breast Cancer. Clinical breast cancer. 2013.

25. Swain SM, Kim SB, Cortes J, Ro J, Semiglazov V, Campone M, Ciruelos E, Ferrero JM, Schneeweiss A, Knott A, Clark E, Ross G, Benyunes MC, Baselga J. Pertuzumab, trastuzumab, and docetaxel for HER2-positive metastatic breast cancer (CLEOPATRA study): overall survival results from a randomised, double-blind, placebo-controlled, phase 3 study. The lancet oncology. 2013; 14:461-471. 